\title{
Modeling the Transient Response of the Thermosyphon Heat Pipes
}

\author{
B. Rashidian, M. Amidpour and M. R. Jafari Nasr
}

\begin{abstract}
This paper presents a theoretical investigation of the thermosyphon heat pipe behavior in transient regime. We take advantage of the transient lumped model to simulate the response of the heat pipe. The transient thermal behavior of the heat pipe has been developed in order to obtain an analytic expression of the system response. Equations solved by Matlab and the models have been performed by Simulink. A computer simulation program based on the lumped method was developed to estimate temperature of the heat pipe as well as the time needed to reach steady state condition. This program can be considered as a simple tool for modeling and designing heat pipe in transient regime. The results from this model were found to be in general agreement with the other numerical models.
\end{abstract}

Index Terms - Heat pipe, Lumped model, Modeling, Response time.

\section{INTRODUCTION}

Heat pipes are widely used for heat recovery and energy saving in various ranges of applications because of their simple structure, special flexibility, high efficiency, good compactness and excellent reversibility. The advantage of using heat pipe over other conventional methods is that large quantities of heat can be transported through small cross section area over a considerable distance with no additional power input to the system. A two-phase closed thermosyphon (TPCT) is passive high performance heat transfer device. It is a closed container filled with a small amount of a working fluid. In such a device, heat is supplied to the evaporator wall, which causes the liquid contained in the pool to evaporate. The generated vapor then moves upwards to the condenser. The heat transported is then rejected into the heat sink by a condensation process. The condensate forms a liquid film which flows downwards due to gravity. Fig. 1 illustrates a conventional heat pipe with three sections: (1) the evaporator section where heat is added to the system; (2) the condenser section where heat is removed from the system; (3) the transport section which connect the evaporator and the condenser, serving as a flow channel.

The working fluid flows inside a heat pipe can be divided

Manuscript received April 28, 2008.

Babak Rashidian is M. Sc. Student at the Department of Mechanical Engineering, K. N. Toosi University of Technology, Tehran, Iran (phone: +98-912-2044418; e-mail: babakrashidian@ gmail.com).

M. Amidpour is Associated Professor at the Department of Mechanical Engineering, K. N. Toosi University of Technology, Tehran, Iran (e-mail: Amidpour@kntu.ac.ir).

M. R. Jafari Nasr is Associated Professor at the Petrochemical Research and Technology Company (npc-rt), Tehran, Iran (e-mail: M.jafarinasr@npc-rt.ir). into four components: (1) vapor flow in the transport section; (2) liquid flow in the transport section; (3) vapor flow in the evaporator and condenser; and (4) liquid flow in the evaporator and condenser.

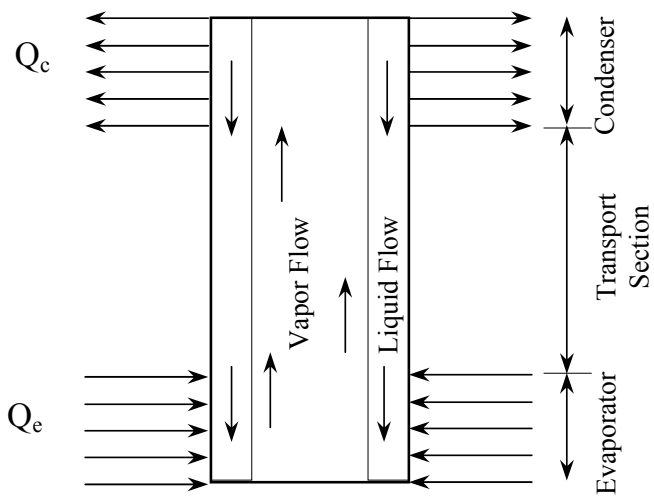

Fig. 1. A sketch of a heat pipe in operation.

The main advantage of TPCTs is that no mechanical pumping is needed. As a consequence, they are cheap and reliable. After a heat pipe reached a fully steady state condition, where the vapor flow is in the continuum state, it is often desirable to determine the temperature and the length of time needed to reach another steady state, for a given new operating conditions. The main investigations performed on TPCT behavior in transient regimes comprise several features. First, theoretical and numerical models describing the behavior of the whole system in response to a step of heat rate applied to the evaporator wall, have been developed by Harley and Faghri [1] and Reed and Tien [2]. It must be emphasized that these models have not been validated during the transient regime because of the lack of experimental data. A second feature deals with the difficulty of modeling unsteady pool boiling regimes occurring in the evaporator such as intermittent boiling, pulse boiling or geyser effect.

Reed and Tien [2] presented a theoretical investigation of the TPCT response time with a model describing the behavior of the whole system in both transient and steady regimes. The authors indicate that, for most TPCTs, the governing time scale was the "film residence time". The authors correlated the response time of the TPCT with the dimensionless parameters: Gr, Bo, Ja, $\rho_{\mathrm{v}} / \rho_{1}$ and $\gamma$. Thus, the TPCT response time can be reduced in various ways: by lowering the thermal inertia of the working fluid, by acting on parameters which play a part in the expression of the "residence film time": Gr, Bo, or by increasing the ratio $\rho v / \rho l$ which make vapor and liquid flow more intense.

In this paper, an analysis of the heat pipe response time has been presented with the aim of providing design advice to minimize this quantity. The transient behavior has been 
investigated, when a sudden change in operating condition is applied to the heat pipe. A mathematical model based on the lumped model [3] presented. Then, the dependence of the heat pipe response time to several parameters has been analyzed.

\section{TRANSIENT LUMPED MODEL}

After a heat pipe reached a fully steady-state condition, where the vapor flow is in the continuum state, it is often desirable to determine the length of time needed to reach another steady state for a given increase in the heat input. Although the 2-D numerical model is generally more comprehensive and accurate, it usually requires considerable time and effort for computer coding. The lumped analytical model, on the other hand, provides a quick and convenient tool for the heat pipe designer. Faghri and Harley [3] presented a transient lumped heat pipe model which determines the average temperature as a function of time.

This formulation is derived from the general lumped capacitance analysis [3], which is an application of an energy balance over a control volume, as shown in Fig. 2. The general lamped analysis results in the following energy equation for the case of both radiative and convective heat transfer from the condenser surface, and an imposed heat input to the evaporator.

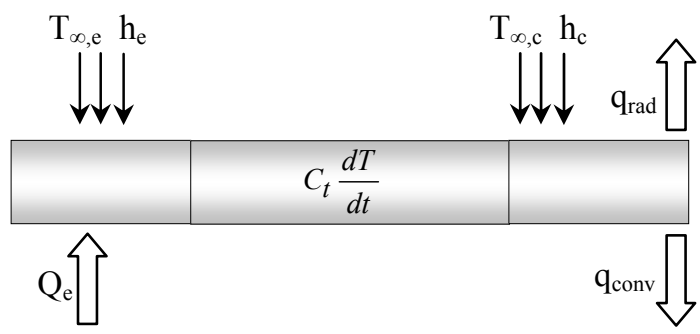

Fig 2. Control volume for lumped capacitance analysis.

$$
\begin{aligned}
& Q_{e}-\left(q_{c o n v}+q_{r a d}\right) S_{c}=C_{t} \frac{d T}{d t} \\
& C_{t}=\rho V_{t} c_{p}
\end{aligned}
$$

where $Q_{e}$ is the heat input, $q_{c o n v}$ is the output heat flux by convection, $q_{r a d}$ is the radiation heat flux, $S_{c}$ is the surface area around the circumference of the cooled section, $C_{t}$ is the total thermal capacity of the system, $T$ is the assumed uniform temperature of the heat pipe, $\rho$ is the density, $V_{t}$ is the total volume, and $c_{p}$ is the specific heat of the system.

However, in heat pipe applications, the total thermal capacity is defined as the sum of the heat capacities of the solid and liquid components of the heat pipe. The heat capacity of the liquid saturated wick is modeled accounting for both the liquid in the wick and the wick structure itself.

$$
\left(\rho c_{p}\right)_{\text {eff }}=\varphi\left(\rho c_{p}\right)_{l}+(1-\varphi)\left(\rho c_{p}\right)_{s}
$$

where $\varphi$ is the prosity of the wick.

Typically, the thermal capacity of the vapor phase is neglected since the mass of vapor is small compared to that of the pipe wall or liquid working fluid. The heat rejection terms due to convection or radiation are found from the external heat transfer coefficients and temperature differences.

$$
Q=q_{\text {conv }} S_{c}=h S_{c}\left(T-T_{\infty, c}\right)
$$

or

$Q=q_{\mathrm{rad}} S_{c}=\varepsilon \sigma S_{c}\left(T^{4}-T_{\infty, c}^{4}\right)$

where $T_{\infty, c}$ is the ambient temperature surrounding the condenser, $h$ is the external convective coefficient, $\varepsilon$ is the emissivity, and $\sigma$ is the Stefan-Boltzmann constant " $5.67 \times 10^{-8} \mathrm{~W} / \mathrm{m}^{2} . \mathrm{K}^{4}$."

For a convective boundary condition, a closed-form solution can be obtained. However, for a radiative boundary, a simple first-order ordinary differential equation must be solved iteratively. To simplify the formulation for a convective boundary condition, two external thermal resistances arc defined as:

$$
\begin{aligned}
& R_{c}=\frac{1}{h_{c} S_{c}} \\
& R_{e}=\frac{1}{h_{e} S_{e}}
\end{aligned}
$$

$S_{e}$ is the surface of the evaporator section, and $h$ is the external convective coefficient at the condenser or evaporator.

In a traditional lumped capacitance analysis, is it very important to verify that the Biot number of the system is less than 0.1 , where the Biot number is a measure of the temperature drop within the system as compared to the temperature drop between the system and the fluid. However, the effective thermal conductivity of a heat pipe is very high, which results in a nearly isothermal temperature profile. In most cases, the effective thermal conductivity of the heat pipe is several orders of magnitude larger than the heat transfer coefficient between the condenser and the environment, and thus the Biot number criterion is satisfied [4], [5]. Several conditions are examined, and closed-form solutions are derived for a convective boundary, which describe the transient lumped temperature of the heat pipe. For the case of Pulsed heat input to the evaporator, constant condenser ambient temperature:

$Q_{e}=\left\{\begin{array}{ll}Q_{e} & \mathrm{t} \prec 0 \\ Q_{e 2}=f(t) & \mathrm{t} \geq 0\end{array}\right.$ and $T_{\infty, c}=T_{\infty, c 1}=$ constant

To determine the initial conditions, the heat pipe is assumed to be at the steady state at $(t<0)$, such that the general lumped capacitance equation simplifies to:

$Q_{e 1}=\frac{T-T_{\infty, c 1}}{R_{c}}=0$

which gives the initial heat pipe temperature as:

$T(0)=R_{c} Q_{e 1}+T_{\infty, c 1}$

At $t=0$, the heat input pulses to $Q_{e}=Q_{e 2}$, and the general lumped capacitance equation is given by:

$Q_{e 2}-\frac{\left(T-T_{\infty, c 1}\right)}{R_{c}}=C_{t} \frac{d T}{d t}$

This is a first order differential equation. The responses are shown in Figs. 3,4 and the simulink [6] model is shown in Fig. 5.

Equation (1) can be solve for various boundary conditions, in example for the case of Pulsed evaporator ambient temperature, constant condenser ambient temperature: 


$$
T_{\infty, e}=\left\{\begin{array}{ll}
T_{\infty, e l} & \mathrm{t} \prec 0 \\
T_{\infty, e 2}=f(t) & \mathrm{t} \geq 0
\end{array} \text { and } T_{\infty, c}=T_{\infty, c 1}=\right.\text { constant }
$$

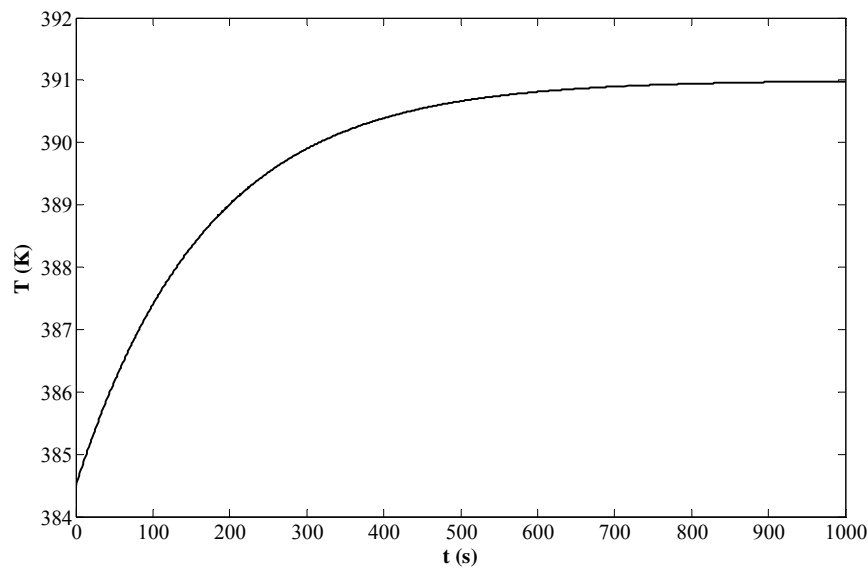

Fig. 3. Heat pipe response to pulsed heat input to the evaporator $\left(Q_{e 2}=\right.$ const., $\left.Q_{e 2}>Q_{e l}\right)$.

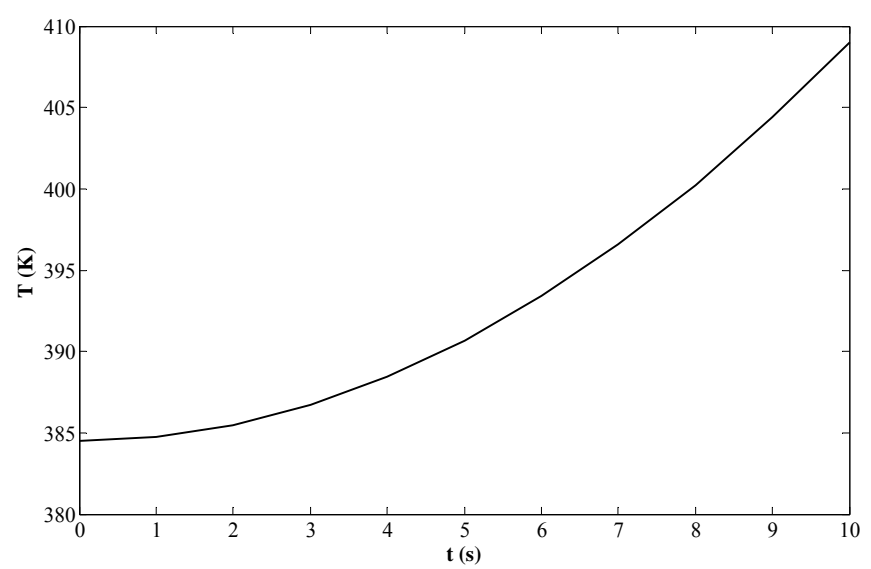

Fig. 4. Heat pipe response to $Q_{e 2}=Q_{e l}(t+1)$.

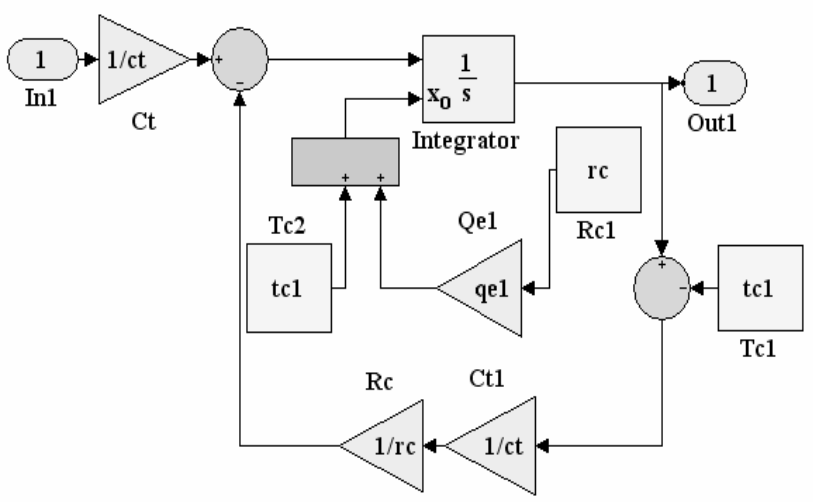

Fig. 5. Simulink model for lumped model. Pulsed heat input to the evaporator, constant condenser ambient temperature.

In this case, the ambient temperature at the evaporator is a function of time, and the transient heat input is found using the thermal resistance between the evaporator and the environment. The initial condition is:

$T(0)=\frac{T_{\infty, e l} R_{c}+T_{\infty, c 1} R_{e}}{R_{c}+R_{e}}$

The energy equation is:
$\frac{\left(T_{\infty, e 2}-T\right)}{R_{e}}-\frac{\left(T-T_{\infty, c 1}\right)}{R_{c}}=C_{t} \frac{d T}{d t}$

The exact solution for $T_{\infty, e 2}=$ constant is given by:

$T(t)=\frac{T_{\infty, c 1} R_{e}+T_{\infty, e 1} R_{c}}{R_{c}+R_{e}}+\frac{\left(T_{\infty, e 2}+T_{\infty, e l}\right)}{R_{c}+R_{e}}\left(1-e^{\frac{-t}{\tau}}\right)$

Where the time constant is given by:

$\tau=\frac{C_{t} R_{c} R_{e}}{R_{c}+R_{e}}$

The simulink model for this case is shown in Fig. 6 and response is shown in Fig. 7.

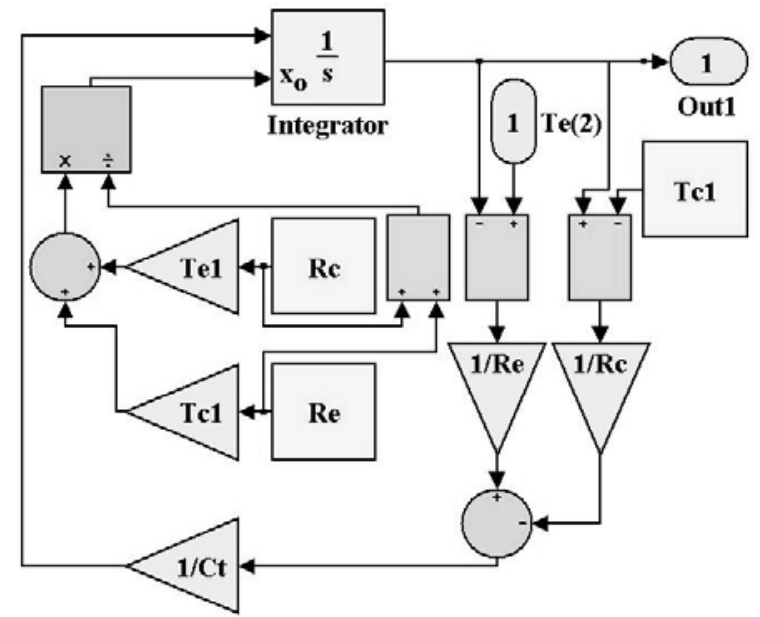

Fig. 6. Simulink model for lumped model. Pulsed evaporator ambient temperature, constant condenser ambient temperature.

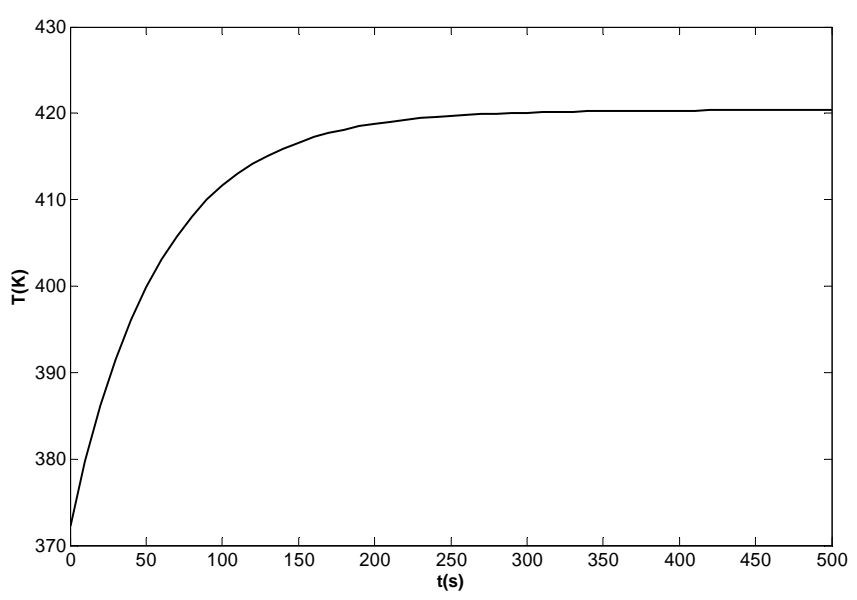

Fig. 7. Heat pipe response to pulsed evaporator ambient temperature $\left(T_{e 2}=\right.$ constant, $\left.T_{e 2}>T_{e l}\right)$.

In the case of constant heat input to the evaporator, pulsed condenser ambient temperature:

$T_{\infty, c}=\left\{\begin{array}{ll}T_{\infty, c 1} & \mathrm{t} \prec 0 \\ T_{\infty, c 2}=f(t) & \mathrm{t} \geq 0\end{array}\right.$ and $Q_{e}=Q_{e 1}=$ constant 
The initial condition is:

$T(0)=R_{c} Q_{e 1}+T_{\infty, c 1}$

and the general lumped equation is:

$Q_{e 1}-\frac{\left(T-T_{\infty, c 2}\right)}{R_{c}}=C_{t} \frac{d T}{d t}$

In the case of constant evaporator ambient temperature, pulsed condenser ambient temperature:

$T_{\infty \propto, c}=\left\{\begin{array}{ll}T_{\infty \propto, c 1} & \mathrm{t} \prec 0 \\ T_{\infty, c 2}=f(t) & \mathrm{t} \geq 0\end{array}\right.$ and $T_{\infty, e}=T_{\infty, e l}=$ constant

In this case, the condenser ambient temperature changes at $t$ $=0$, while the evaporator ambient temperature remains constant. The initial temperature is:

$$
T(0)=\frac{\left(T_{\infty, e 1} R_{c}+T_{\infty, c 1} R_{e}\right)}{R_{e}+R_{c}}
$$

The general lumped capacitance equation for $t>0$ is:

$\frac{T_{\infty, e 1}-T}{R_{e}}-\frac{T-T_{\infty, c 2}}{R_{c}}=C_{t} \frac{d T}{d t}$

Fig. 8, represents response of the system to pulsed condenser ambient temperature.

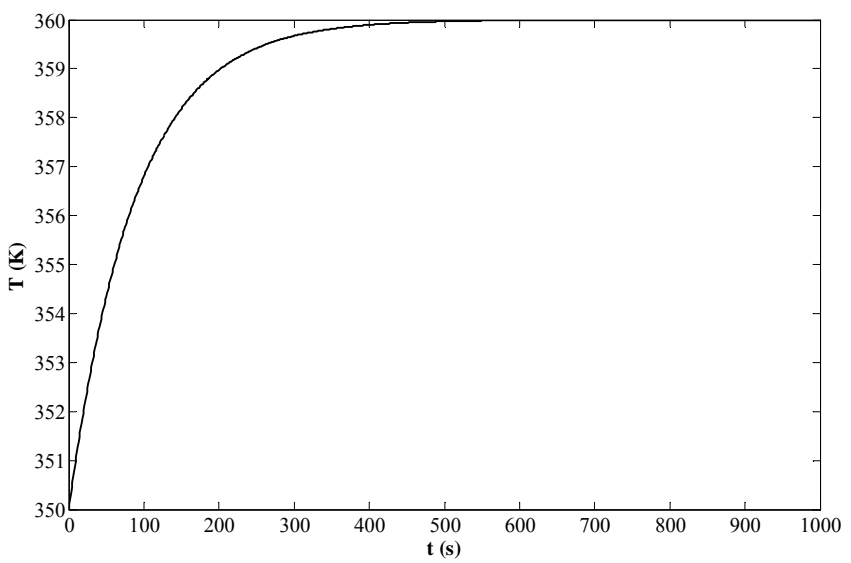

Fig. 8. Response to pulsed condenser ambient temperature.

For the case of radiative heat transfer at the condenser, with a constant ambient condenser temperature and pulsed heat input to the evaporator, the general lumped capacitance equation becomes:

$\frac{d T}{d t}+\frac{\varepsilon \sigma S_{c} T^{4}}{C_{t}}=\frac{Q_{e 2}}{C_{t}}+\frac{\varepsilon \sigma S_{c} T_{\infty, c 1}^{4}}{C_{t}}$

Equation (23) is a first-order, nonlinear, nonhomogeneous, ordinary differential with no closed form analytical solution. However, a numerical solution can be found using the Newton/Raphson secant technique. Using the same procedure as in the convective cases above, the initial temperature can be found as:
$T_{0}=\left(\frac{Q_{e 1}}{\varepsilon \sigma S_{c}}+T_{\infty, c 1}^{4}\right)^{1 / 4}$

The contribution of radiation from the environment to the heat pipe is included in the formulation, but in most cases has a very small effect on the temperature profile and can be neglected.

\section{MATLAB PROGRAM}

To analysis response of the heat pipe the program "Heat Pipe Response" has been developed by Matlab. The input arguments are: physical properties of the heat pipe wall $(\mathrm{Cp}$, Thickness, Length of the heat pipe, Fill ratio, ...), Physical properties of the working fluid and properties of the wick.

Program estimate heat pipe response for these cases:

1- Constant condenser ambient temperature, changing of the heat input to the evaporator.

2- Constant heat input to the evaporator, changing of the condenser ambient temperature.

3- Constant condenser ambient temperature, changing of the evaporator ambient temperature.

4- Constant evaporator ambient temperature, changing of the condenser ambient temperature.

5- Constant ambient condenser temperature, with radiative heat transfer at the condenser, changing of the heat input to the evaporator.

The change of the operating condition are pulse, linear and exponential, in the case of pulsed input, program calculate time to reach the new steady state condition and the temperature of the new steady state condition. Some shots of the graphical user interface of the program are shown in fig. $9,10,11$, and 12 .

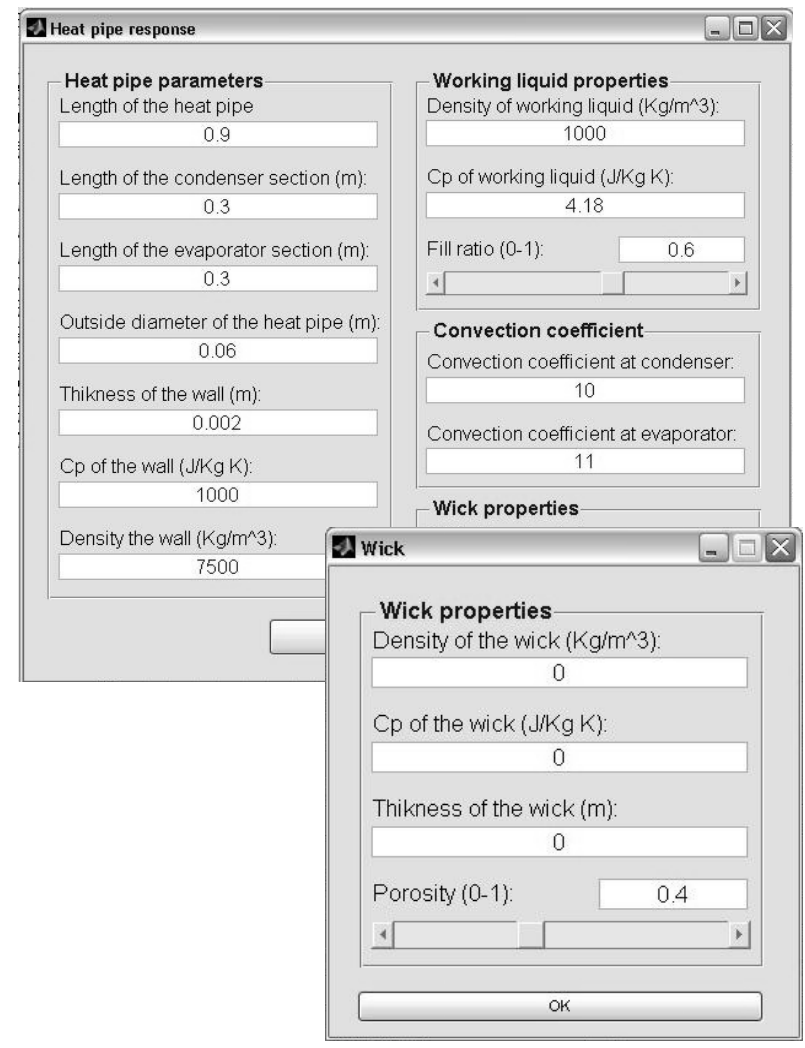

Fig. 9. First page of the "Heat pipe respose" program. 


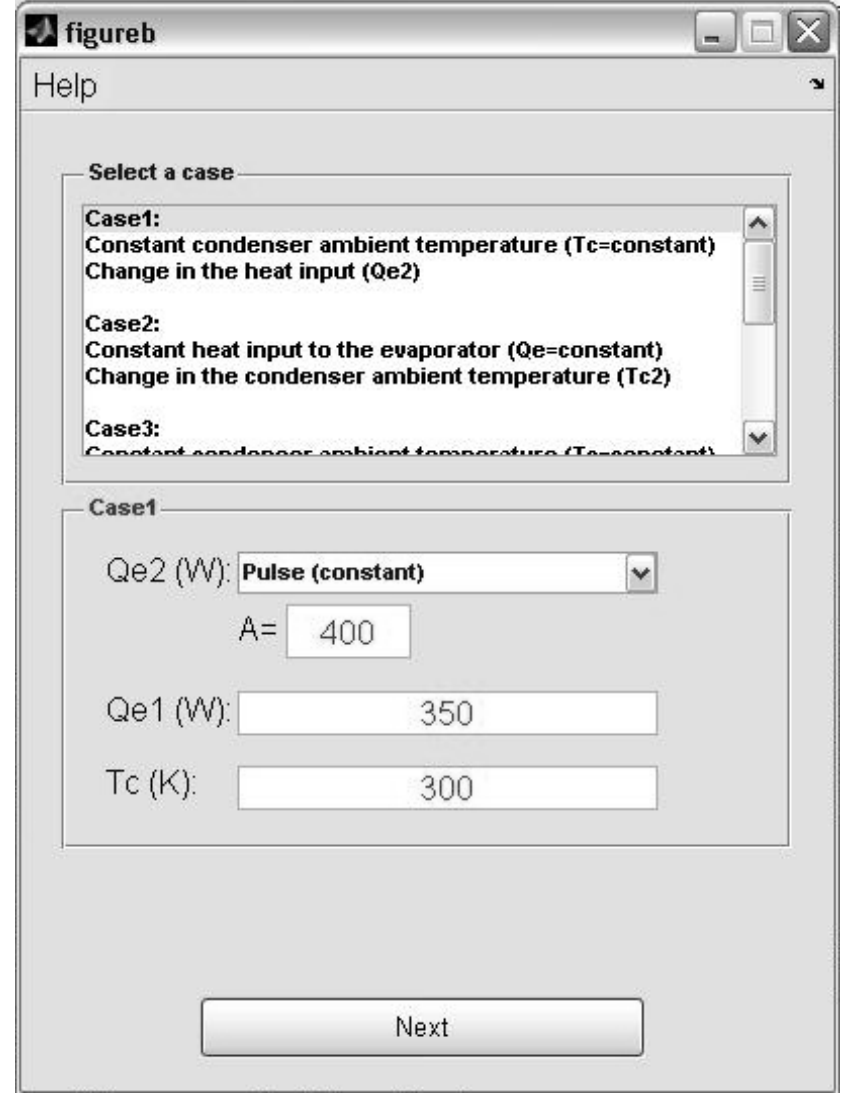

Fig. 10. Case selecting and assigning input type.

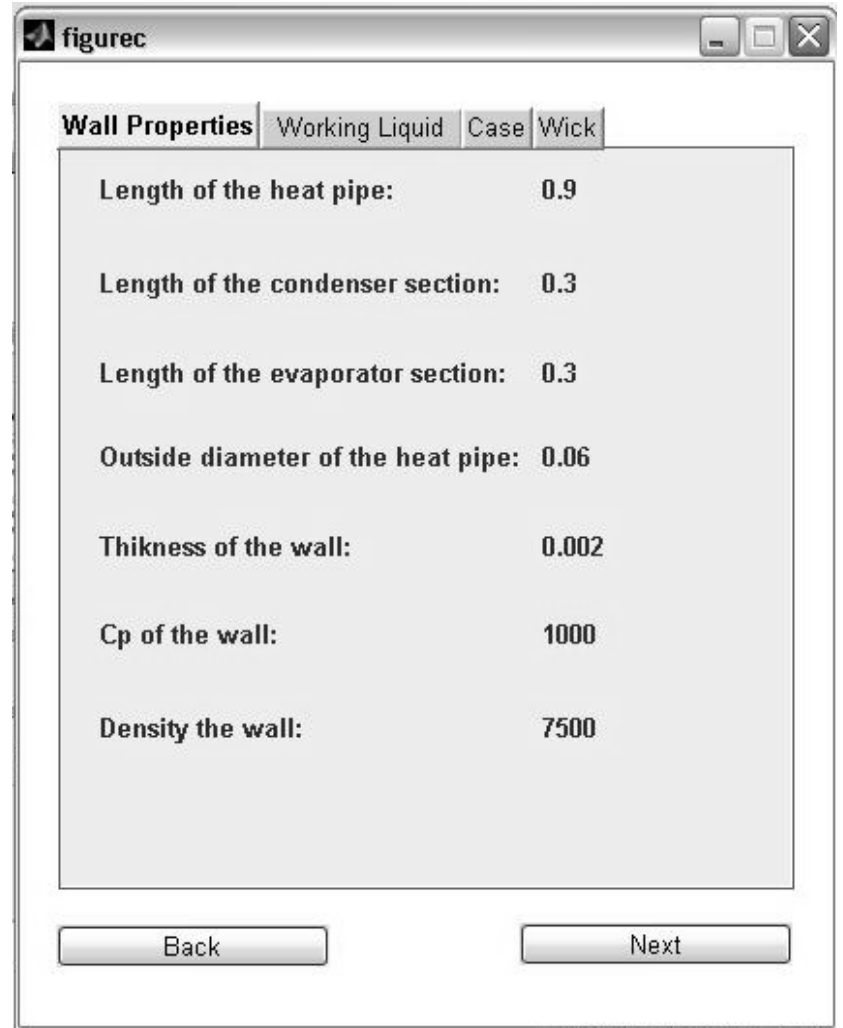

Fig. 11. Verifying data.

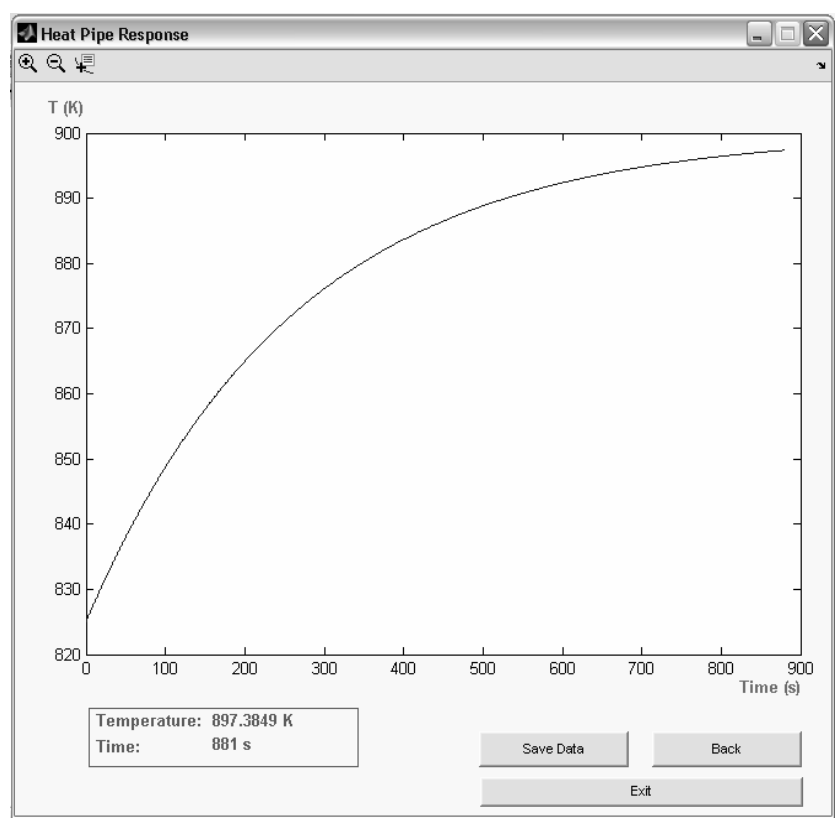

Fig. 12. Results.

\section{RESULTS DISCUSION}

Fig. 13, represents validation of the lumped capacitance analysis. The results of the lumped model with a pulsed heat input and a constant condenser ambient temperature (Equation 11) were compared to the numerical analysis for a sodium heat pipe with a stainless steel wall having a total heat capacity of $\mathrm{C}_{\mathrm{t}}=175.4 \mathrm{~J} / \mathrm{K}[3]$.

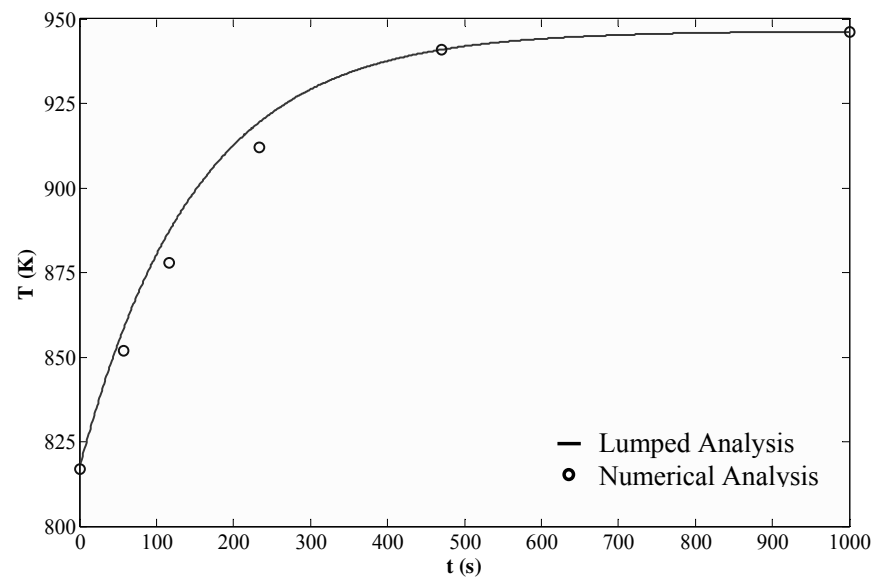

Fig.13. Transient temperature profile for the lumped capacitance model as compared with the numerical analysis.

\section{CONCLUSION}

In this paper, a mathematical model has been constructed for thermosyphon heat pipe, in order to describe thermal behavior in transient regime. An influence analysis of the heat pipe response to various operating conditions has been shown. A computer program has been developed to simulate heat pipe in transient regime, this program can be use for heat pipes with capillary media as well as wickless heat pipes. 
Proceedings of the World Congress on Engineering 2008 Vol II WCE 2008, July 2 - 4, 2008, London, U.K.

\section{REFERENCES}

[1] C. Harley, A. Faghri, "Complete transient two-dimensional analysis of two-phase closed thermosyphons including the falling condensate film," Transactions of the ASME 116, 1994, 418-26.

[2] J.G. Reed, C.L. Tien, "Modeling of the two-phase closed thermosyphon," Transactions of the ASME 109, 1987, 722-730.

[3] Faghri, A, "Heat Pipe Science And Technology," Taylor \& Francis, USA., 1995.

[4] Dunn, P., D. and Reay, D., A., “ Heat Pipe, " $4^{\text {th }}$ Edition, Pergamon Press, 1994.

[5] Peterson, G. P., “An Introduction To Heat Pipes,” John Wiley \& Sons, 1994.

[6] Karris, T., S., "Introduction To Simulink With Engineering Applications, "Orchard Pub, 2006. 